\title{
Spinal Cord Stimulation for the Management of Refractory Neuropathic Pain in Patients with Spinal Dysraphism, Case Series of 4 Patients with 44 to 53 Month Follow Up
}

\author{
Jonathan*, Gavin Quigley and Peter Mcgarity \\ School of Pharmacy, Centre for Advanced and Interdisciplinary Radiation Research, UK
}

Submission: March 01, 2018; Published: August 24, 2018

*Corresponding author: Jonathan, School of Pharmacy, Molecular Therapeutics, Centre for Advanced and Interdisciplinary Radiation Research, UK, Email: jpoots01@qub.ac.uk

Abstract

Objectives: Northern Ireland has a high incidence of spinal dysraphism and a significant adult population with a history of multiple previous surgical interventions for the condition. Spinal cord stimulation (SCS) is widely used for neuropathic leg pain but rarely in patients with spinal dysraphism, perhaps because of technical concerns regarding insertion and placement of epidural leads. We report the use spinal cord stimulation for the management of neuropathic pain successfully in three patients with tethered cords that had previously undergone surgical interventions and failed maximal medical management of their chronic neuropathic pain, we also report on one unsuccessful case.

Materials and methods: This were a retrospective review of the medical records for 4 cases with prospective follow up.

Results: Three patients showed ongoing benefit with follow up of up to 53 months, one patient experienced no benefit from the procedure.

Conclusion: This case series adds to the body of evidence that spinal cord stimulation can be used effectively and safely in cases of neuropathic pain related to spinal dysraphism.

Keywords: Chronic neuropathic pain; Spinal dysraphism; Surgical interventions; Spinal cord

\section{Introduction}

Tethered cord syndrome is typically the result of a developmental abnormality where the conus of the spinal cord is attached to the vertebral column, making it susceptible to abnormal prolonged stretching. Our understanding of the pathophysiology of tethered cord syndrome mainly comes from animal models. It has been shown in feline models that increased traction on the filum terminal results in blood flow and oxidative metabolism becoming impaired.

Yamada et al. [1] showed that the degree of cord dysfunction and reversibility correlated with the duration and magnitude of traction. 1 Traction of the filum appears to have a greater effect on the more caudal segments of the cord as opposed to the more rostral segments and this is partly due to the dentate ligament having a protective effect on the rostral segments. Kocak et al., [2] showed that fixing the filum terminal to the sacrum and surrounding tissue resulted in ischemic injury with reduced motor and sensory nerve conduction.
Tethered cord is usually associated with a form of spinal dysraphism and is especially common in patients with spinabifida following the closure of the myelomeningocele. Symptoms associated with tethered cord syndrome are classically lower limb neurology in the form of both weakness and sensory changes, bowel and bladder control dysfunction, gait abnormalities, musculoskeletal deformity and lower back/perineal/lower limb neuropathic pain. Early surgical intervention with an aim to release the tethering and therefore release the tension on the cord is indicated for patients with new onset/progressive neurology and has been shown to improve symptoms, especially pain [3].

A retrospective study of clinical outcomes after neurosurgical intervention in 60 adults with primary treatment of tethered cord syndrome showed at follow up 78\% had improved back and $83 \%$ improved leg pain [4]. This does mean approximately 1 in 5 patients who undergo surgical untethering will have refractory pain that is notoriously hard to treat with conventional analgesia 
They also remain at risk of recurrent tethering due to scarring following any untethering procedure. Spinal cord stimulation offers an option for management of pain for these patients without the risks associated with manipulation of neural tissues at a previously operated site which may lead to further neurological disability. Patients with recurrent tethering have a worse prognosis with many developing permanent neurological deficits and intractable pain [5]. We propose that spinal cord stimulation can be used effectively in the management of neuropathic lower limb pain that is refractory to other medical/ surgical treatment modalities.

The use of spinal cord stimulation for control of neuropathic pain is based on the "gate control theory" first presented by Melzack and Wall in the 1960's [6]. The dorsal horns of the spinal cord contain large afferent nerve fibres and by applying electrical stimulation to these A-beta fibers you can inhibit nociceptive transmission from that segmental level. It is also thought that inhibition of afferent activity in the spinothalamic tracts, long-term suppression of sympathetic activity and antidromic effects on peripheral reflex circuits may take part in the pain alleviation $[7,8]$.

The electrodes from spinal cord stimulator tend to be placed at the level of T9/T10 in order to stimulate the patient's whole leg and lower back. The electrodes are either implanted via laminectomy or can be inserted percutaneously. Electrodes that are inserted via laminectomy have been shown to have a significantly greater long-term pain relief as opposed to percutaneous electrodes. They have also been shown to have lower stimulation requirements and are less likely to migrate [9].

Although the use of spinal cord stimulation for the management of neuropathic pain is well established a literature review regarding its use in patients with pain secondary to tethered cord syndrome is limited. Our literature search highlighted two previous case reports describing its use in two adult patients Moens, et al. [10] \& Tyagi et al. [11] Both of which reported a significant benefit in the management of pain for these patients. Moens reported its usage in a 37-year-old female patient with neuropathic pain following a history 14 years previously of untethering her spinal cord and excision of a sacral myelomeningocele with a single lead inserted at T12. Tyagi et al. [11] reported its usage in 19-year-old patient that had a cord untethering procedure for a lipomyelomeningocele at the age of 12 and then again at the age of 19 . They inserted two leads at the level of $\mathrm{T} 8$.

\section{Case presentation}

We present here the cases of four patients with a history of tethered cord syndrome secondary to dysraphism of the spine who have had spinal cord stimulation to manage lower limb neuropathic pain. Each patient had previously undergone a surgical procedure for the spinal abnormality and were considered unsuitable for any further spinal surgery. Their pre- operative workup included an assessment of the patient's pain with use of a visual analogue scale scoring the pain in severity from 1 (mild) to 10 (severe). All patients had a preoperative MRI of their spine and the spinal cord stimulators were initially inserted for a trial period of between three to seven days. The third patient experienced a more complicated post-operative course and required a switch to high density stimulation which is also discussed. Three patients have now had between 44 and 53 months follow up with ongoing symptomatic relief. One patient did not find the stimulation therapeutic and had the stimulator removed after a brief period.

Patient A - A 39-year-old lady with complex spinal dysraphism in the form of diastematomyelia with refractory neuropathic pain in her right leg and urinary frequency. She had undergone a failed attempt to untether her cord 14 years previous and did not want a further attempt to untether her cord. She underwent a T10/T11 laminectomy and insertion of a spinal cord stimulator (2x8 Specify Medtronic Inc). She was discharged home for a five-day trial period. She found the spinal cord stimulator very effective in relieving her pain, her visual analogue score went from nine to four, with excellent coverage over both legs. She retained full muscular power in both legs and she had normal urinary continence with a subjective improvement in her urinary frequency. She has been followed up for 44 months with ongoing therapeutic benefit. She uses the device continuously and describes its effects as extremely beneficial but finds it difficult to rate the effectiveness on a visual analogue scale (Figure 1).
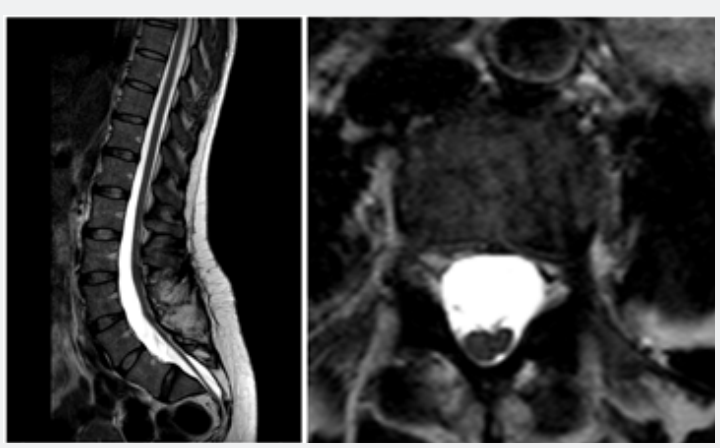

Figure 1: 39-year-old lady with complex spinal dysraphism in the form of diastematomyelia with refractory neuropathic pain in her right leg and urinary frequency.

Patient B - This was a 29-year-old male with spina-bifida which was closed as a young infant. He had the expected Chiari II malformation but also an element of spinal scoliosis and a ventriculo-peritoneal shunt in-situ with multiple previous revisions to the VP shunt system. He complained of worsening neuropathic pain in his right leg over the previous six years that had proven refractory to maximal medical pain management. A percutaneous trial lead was inserted with the tip at the level of T9 (Vectris Medtronic Inc). This resulted in an immediate improvement in his leg pain and internalization was performed 


\section{Open Access Journal of Neurology \& Neurosurgery}

seven days later. He has now been followed up for 44 months with continued effect pain reduction. He uses the device daily and would rate his pain 2 out of 10 on a visual analogue scale when the stimulation is on (Figure 2).

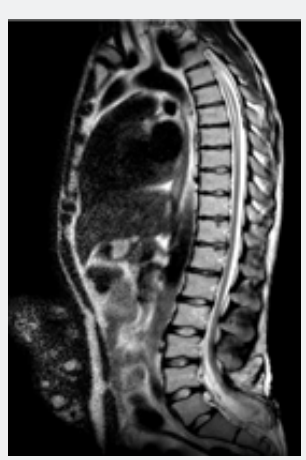

Figure 2: This was a 29-year-old male with spina-bifida which was closed as a young infant.

Patient C - This was a 17-year-old female patient with previous untethering of the cord and decompression of an intradural lipoma at the age of nine. Following the untethering of the cord in 2004 her gait improved however she had persistent allodynia in the sole of her right foot and burning dysasthesia of her anterior right thigh which had remained refractory to maximal medical pain management. Post-operative MRI imaging confirmed satisfactory decompression of the spinal cord and no further exploration of this area was felt to be likely beneficial, so she was referred for consideration of spinal cord stimulation (Figure 3).

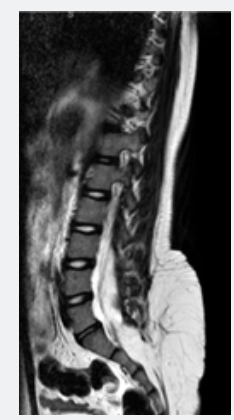

Figure 3: This was a 17-year-old female patient with previous untethering of the cord and decompression of an intradural lipoma at the age of nine.

During the insertion of the spinal cord stimulator she had a laminectomy performed at L3/L4 through an incision in her old scar and the tip of the trial lead was placed at the level of T10 (Vectris Medtronic Inc). The trial of stimulation showed a good response with a reduction in her visual analogue score of $>50 \%$ and the lead was internalized after a trail period of five days.

Unfortunately, at six months post spinal cord stimulator insertion the patient sustained a fall and as a consequence the spinal cord stimulator stopped working with complete loss of coverage. She required further surgery for lead replacement which was successful, with good coverage and pain relief re- established, the previous percutaneous lead was replaced with a paddle lead (2x8 Specify Medtronic Inc).

However, the effectiveness of the stimulation began to reduce, and this resulted in a need to increase her output current. She was scheduled for IPG replacement and prior to this had a trial of high density stimulation with settings of $500 \mathrm{~Hz}$ and 500 microseconds at $2 \mathrm{~mA}$. She subsequently had her IPG replaced with a rechargeable implant in June 2015. She chose to continue with high density settings. She is currently experiencing good relief of her leg allodynia and would rate it as 4 out of 10 when the device is on, she had been followed up for over 53 months with continued effect pain reduction, unfortunately she does experience other painful areas that are not covered by the device. Following telephone review, she has been using the device less frequently now that it has been changed to a rechargeable device, due to her active lifestyle she finds it difficult to keep the device charged.

Patient D - Her background history was of spina bifida with a previous untethering her imaging had been reviewed by 2 consultant neurosurgeons who felt further untethering would be unlikely to be beneficial. The patient had a trial spinal cord stimulator inserted on $07 / 05 / 16$ but then had the device removed on 11/05/16 4 days later as she found the stimulation unpleasant and non-therapeutic despite programming (Figure 4).

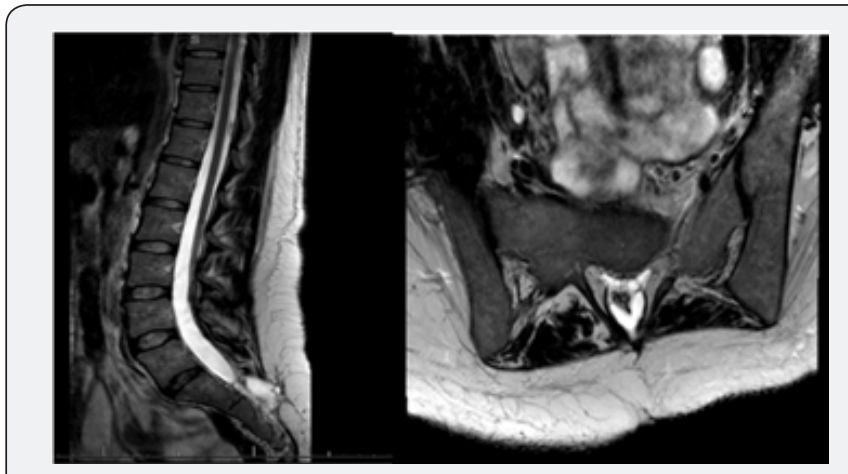

Figure 4: Her background history was of spina bifida with a previous untethering her imaging had been reviewed by 2 consultant neurosurgeons who felt further untethering would be unlikely to be beneficial.

\section{Discussion}

Three of our patients had an immediate and significant benefit from the use of spinal cord stimulation and each patient went on to have the trial leads fully internalized. Despite the significant improvements in pain the patients were still able to perceive normal sensation to hot and cold stimuli along with sharp and soft touch. Due to the stretching of the tethered cord it was necessary to place the electrode more caudally than in patients with a normal spinal cord. This is more problematic in patients with neuropathic leg/buttock pain as it has been shown that in patients with tethered cord syndrome the maximal amount of elongation occurs in the lumbosacral region. 


\section{Open Access Journal of Neurology \& Neurosurgery}

In patients with tethered cord syndrome the spinal cord lies next to the posterior dura either due to attachments or scaring related to previous interventions. This meant that the voltage of stimulation required to achieve satisfactory pain relief was lower than in other non-tethered cord syndrome cases, this is likely to provide a useful reduction in energy consumption and extend battery life. Given the nature of these patients they are likely to require further MRI scans during their lifetime, so the usage of MRI conditional spinal cord stimulator systems is likely prudent.

\section{Conclusion}

Spinal cord stimulation in spinal dysraphism is very effective for management of pain resistant to medical therapy and technical difficulties can be easily overcome. These findings should raise consideration for a randomised multicenter trial of further spinal surgery vs. SCS if there is felt to be clinical equipoise. Now that MRI conditional systems are available the need for a patient to have an MRI in the future should no longer be considered a contra-indication to the management of pain with a spinal cord stimulator.

\section{References}

1. Yamada S (2007) Pathophysiology of tethered cord syndrome and similar complex disorders. Neurosurg Focus 23(2): E6.

2. Kocak A, Kilic A, Nurlu G, Konan A, Kilinic K, et al. (1997) A new model for tethered cord syndrome: a biochemical, electrophysiological and electron microscopic study. Paediatric Neurosurgery 26(3): 120-126.
3. Lew SM (2007) Tethered cord syndrome: an updated review. Paediatric Neurosurgery 43(3): 236-248.

4. Lee GY, Paradiso G, Tator CH, Gentili F, Massicotte EM, et al. (2006) Surgical management of tethered cord syndrome in adults: indications, techniques, and long-term outcomes in 60 patients. J Neurosurg Spine 4(2): 123-131.

5. Klekamp J (2011) Tethered cord syndrome in adults. J Neurosurg Spine 15(3): 258-270.

6. Melzak R (1999) From the gate to the neuromatrix. Pain (6):S121-S126.

7. Compton AK, Shah B, Hayek SM (2012) Spinal cord stimulation: a review. Curr Pain Headache Rep 16: 35-42.

8. Wu M, Linderoth B, Foreman RD (2008) Putative mechanisms be- hind effects of spinal cord stimulation on vascular diseases: a review of experimental studies. Auton Neurosci 138(1-2): 9-23.

9. Smits H, van Kleef M, Holsheimer J, Joosten EA (2013) Experimental spinal cord stimulation and neuropathic pain: mechanism of action, technicalaspects, and effectiveness. Pain Pract 13(2): 154-168.

10. Moens M, Smedt AD, D’haese J, Droogmans S, Chaskis C (2010) Spinal cord stimulation as a treatment for refractory neuropathic pain in tethered cord syndrome: a case report. J Med Case Rep 4: 74.

11. Tyagi R, Kloepping C, Shah S (2016) Spinal cord stimulation for recurrent tethered cord syndrome in a paediatric patient:case report Journal of Neurosurgery: Paediatrics 18(1): 105-110.

\section{Your next submission with Juniper Publishers} will reach you the below assets

- Quality Editorial service

- Swift Peer Review

- Reprints availability

- E-prints Service

- Manuscript Podcast for convenient understanding

- Global attainment for your research

- Manuscript accessibility in different formats

( Pdf, E-pub, Full Text, Audio)

- Unceasing customer service

Track the below URL for one-step submission https://juniperpublishers.com/online-submission.php 